\title{
Additionsreaktionen von 2-Thiazolin-5-thionen mit Acetylenen
}

\author{
Jenny, Christjohannes ; Obrecht, Daniel ; Heimgartner, Heinz
}

\begin{abstract}
Addition Reactions of 2-Thiazolin-5-thiones with Acetylenes. The reaction of the 2-thiazolin5-thiones 1 and 5, respectively, with acetylene carboxylates yields mainly 1,4-dithiafulvenes of type 3 . In the presence of the acetylenic compounds, the 1,4-dithiafulvenes undergo an isomerization to give the corresponding 2,3-dihydrothiophene-2-thiones 4 .
\end{abstract}

DOI: https://doi.org/10.1002/hlca.19820650829

Posted at the Zurich Open Repository and Archive, University of Zurich ZORA URL: https://doi.org/10.5167/uzh-97545 Journal Article

Originally published at: Jenny, Christjohannes; Obrecht, Daniel; Heimgartner, Heinz (1982). Additionsreaktionen von 2-Thiazolin5-thionen mit Acetylenen. Helvetica Chimica Acta, 65(8):2583-2586.

DOI: https://doi.org/10.1002/hlca.19820650829 


\title{
260. Additionsreaktionen von 2-Thiazolin-5-thionen mit Acetylenen
}

\author{
Vorläufige Mitteilung ${ }^{1}$ ) \\ von Christjohannes Jenny ${ }^{2}$ ), Daniel Obrecht und Heinz Heimgartner ${ }^{3}$ ) \\ Organisch-chemisches Institut der Universität Zürich, Winterthurerstrasse 190, CH-8057 Zürich
}

(14.IV.82)

Addition Reactions of 2-Thiazolin-5-thiones with Acetylenes

\section{Summary}

The reaction of the 2-thiazolin-5-thiones $\mathbf{1}$ and $\mathbf{5}$, respectively, with acetylene carboxylates yields mainly 1,4-dithiafulvenes of type 3 . In the presence of the acetylenic compounds, the 1,4-dithiafulvenes undergo an isomerization to give the corresponding 2,3-dihydrothiophene-2-thiones 4.

Vor kurzem haben wir über eine einfache Synthese von 4,4-disubstituierten 2-Phenyl-2-thiazolin-5-thionen berichtet [1]. Im Rahmen von Studien zur Reaktivität dieser bisher nicht untersuchten Verbindungsklasse sind $u$. a. thermische Reaktionen mit Acetylenen, Olefinen und 1,3-dipolaren Verbindungen durchgeführt worden. Einige Ergebnisse der Reaktionen mit Acetylenen sollen hier kurz wiedergegeben werden ${ }^{4}$ ).

Umsetzungen von Acetylenen mit S-Heterocyclen, die das Strukturelement $-\mathrm{C}(\mathrm{S})-\mathrm{S}-$ enthalten, d.h. mit Verbindungen vom Typ a (Schema l), sind von

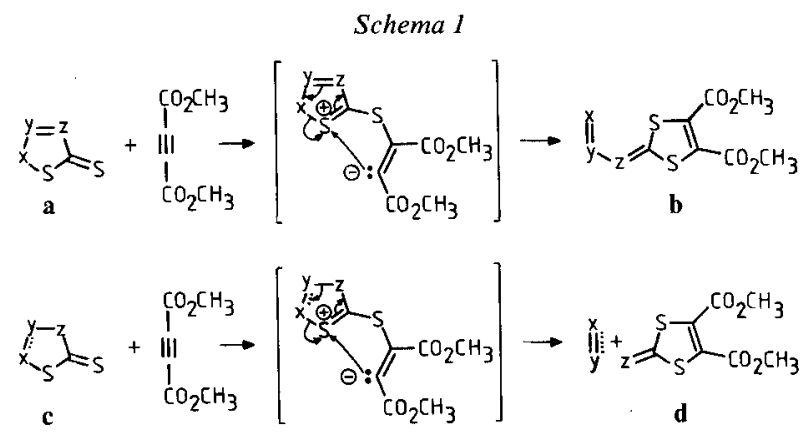

\footnotetext{
1) Eine vollständige Mitteilung soll in dieser Zeitschrift erscheinen.

2) Teil der geplanten Dissertation von Ch. Jenny.

3) Korrespondenzautor.

4) Für 1,3-dipolare Cycloadditionen an 2-Thiazolin-5-thione s. [2].
} 
mehreren Arbeitsgruppen intensiv untersucht worden (vgl. [3-10] und dort zit. Literatur). Als Hauptprodukte wurden im allgemeinen Verbindungen vom Typ b isoliert. Der Mechanismus dieser Reaktionen kann über eine dipolare Zwischenstufe (Schema 1) oder eine unter Ringöffnung verlaufende 1,3-dipolare Cycloaddition formuliert werden.

In Analogie zu der mit a skizzierten Reaktion müsste die Umsetzung von S-Heterocyclen des Typs $\mathbf{c}$ mit Acetylenen unter Fragmentierung zu Verbindungen vom Typ d führen (Schema 1). Tatsächlich ist dieser Reaktionstyp vereinzelt beobachtet worden (z. B. für $x-y-z=N=C(A r)-S$ [11], CHR $-C^{\prime} H R^{\prime}-S$ [4] [6] und $\left.\mathrm{C}(\mathrm{SR})=\mathrm{N}-\mathrm{NR}^{\prime}[7]\right)$.

Die Umsetzung von 4,4-Dimethyl-2-phenyl-2-thiazolin-5-thion (1) mit Acetylendicarbonsäure-dimethylester (2a) in Toluol bei $80^{\circ}$ (Bombenrohr) führt unter Abspaltung von Benzonitril (gas-chromatographischer Nachweis) in $78 \%$ Ausbeute zum 1,4-Dithiafulven 3a (Schema 2). Als Nebenprodukt bildet sich das 2,3Dihydrothiophen-2-thion 4a, das nach chromatographischer Reinigung in $c a$. 2\% Ausbeute erhalten wurde. Das Verhältnis der beiden Produkte ist von der Reaktionstemperatur abhängig, wie dies aus den Resultaten im Schema 2 ersichtlich ist. Auch die Reaktion von 1 mit Propiolsäureäthylester (2b) führt zu Produktgemischen, wobei aber von den beiden Isomeren $\mathbf{3 b}$ und $\mathbf{4 b}$ das letztere deutlich überwiegt (Schema 2).

Kontrollversuche bei $180^{\circ}$ haben ergeben, dass die Produkte 3 und 4 unter diesen Bedingungen stabil sind. So lagert sich z. B. das Dithiafulven 3a rein thermisch nicht in das Dihydrothiophenthion 4a um. In Gegenwart von 2a verläuft diese Isomerisierung bei $180^{\circ}$ jedoch leicht, was am einfachsten mit dem in Schema 3 formulierten Reaktionsmechanismus erklärt werden kann (entsprechend $\mathbf{c} \rightarrow \mathbf{d}$ in Schema I).

Die Charakterisierung und Strukturzuordnung der Reaktionsprodukte erfolgte mit Hilfe der spektroskopischen Daten ${ }^{5}$ ). Dabei sind jeweils neben UV.-, IR.-, ${ }^{1}$ H-NMR.- und Massenspektrum vor allem die ${ }^{13} \mathrm{C}$-NMR.-Daten herangezogen worden ${ }^{6}$ ).

Schema 2
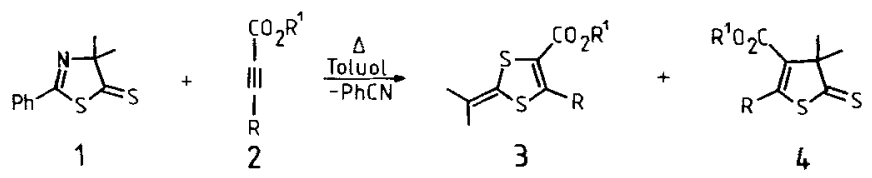

a $\begin{array}{ll}\mathrm{R}=\mathrm{CO}_{2} \mathrm{CH} \\ \mathrm{R}^{1}=\mathrm{CH}_{3}\end{array}$

$80^{\circ}$
$150^{\circ}$
$180^{\circ}$

$3 \mathbf{a} / \mathbf{4 a}$

ca. $30 / 1$

$1 / 1$

$1 / 2$

b $\mathrm{R}=\mathrm{H} \quad 145^{\circ}$ $\mathrm{R}^{1}=\mathrm{CH}_{2} \mathrm{CH}_{3} \quad 170^{\circ}$

$3 \mathbf{b} / \mathbf{4 b}$

ca. $1 / 3$

5) Alle Verbindungen ergaben korrekte Elementaranalysen für $\mathrm{C}, \mathrm{H}, \mathrm{N}$ und $\mathrm{S}( \pm 0,3 \%)$.

$\left.{ }^{6}\right)$ Daten von 3a. - Rötliche Kristalle vom Smp. 58-60. $-{ }^{13} \mathrm{C}-\mathrm{NMR}$. $\left(\mathrm{CDCl}_{3}\right)$ : 160,3 $\left(2 \mathrm{COOCH}_{3}\right)$; $130,7,120,0$, und 119,2 (4 olefin. C-Atome); 53,0 $\left(2 \mathrm{COOCH}_{3}\right) ; 22,7\left(\left(\mathrm{CH}_{3}\right)_{2} \mathrm{C}\right)$. - Daten von 4 a. Rotes Öl, Sdp. $100 \%, 1$ Torr. $-{ }^{13} \mathrm{C}-\mathrm{NMR}$. $\left(\mathrm{CDCl}_{3}\right) ; 243,0(\mathrm{C}=\mathrm{S}) ; 163,5$ und $160,4\left(2 \mathrm{COOCH}_{3}\right)$; 147,6 und 131,6 (2 olefin. C-Atome); 70,6 (C(3)); 53,1 und 52,7 $\left(2 \mathrm{COOCH}_{3}\right) ; 28,3\left(\left(\mathrm{CH}_{3}\right)_{2} \mathrm{C}\right)$. 
Schema 3

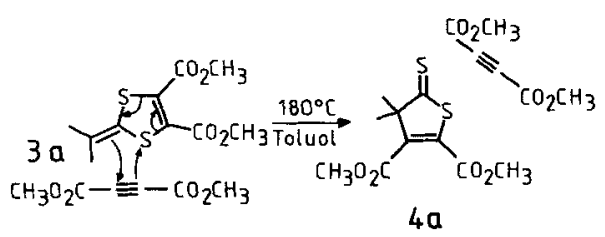

Für die entsprechenden Umsetzungen des 4-Methyl-2,4-diphenyl-2-thiazolin5-thions (5) mit Acetylencarbonsäureestern sind etwas höhere Reaktionstemperaturen erforderlich. Die Reaktionen verlaufen aber insofern einheitlicher, als die Dithiafulvene 3c-e unter den gewählten Bedingungen als einzige Produkte auftreten (Schema 4). Die Ausbeute für 3c beträgt 95\%; die beiden isomeren Dithiafulvene $3 \mathrm{~d}$ und $3 \mathrm{e}$ werden als $(1: 1)$-Gemisch in $53 \%$ Ausbeute erhalten.

Einen Sonderfall stellt die Reaktion von 1 bzw. 5 mit Diphenylacetylen (Tolan) dar, welche erst bei Temperaturen um $300^{\circ}$ abläuft. Neben einer Vielzahl von Nebenprodukten wird als Hauptprodukt Tetraphenylthiophen (6, Schema 4) gebildet, das in Ausbeuten von $70 \mathrm{bzw}$. 75\% isoliert wird. Für die Bildung von 6 kommen mehrere Reaktionswege in Frage, die in einer ausführlichen Mitteilung diskutiert werden sollen ${ }^{7}$ ).

Schema 4

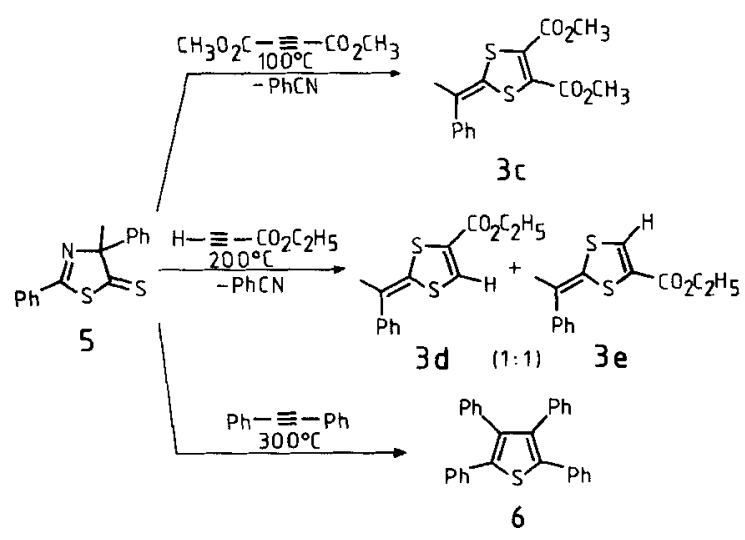

Wir danken den analytischen Abteilungen unseres Institutes für Spektren und Elementaranalysen sowie dem Schweizerischen Nationalfonds zur Förderung der wissenschaftichen Forschung und der F. Hoffmann-La Roche \& $\mathrm{Co}$. AG, Basel, für finanzielle Unterstützung.

7) Tetraphenylthiophen (6) wird beim Erhitzen verschiedener Schwefelverbindungen in Gegenwart von Tolan erhalten (siehe z.B. [12]). 


\section{LITERATURVERZEICHNIS}

[1] D. Obrecht \& H. Heimgartner, Chimica 36, 78 (1982).

[2] D. Obrecht, R. Prewo, J. H. Bieri \& H. Heimgartner, Helv. Chim. Acta 65, 1825 (1982); Th. Büchel \& H. Heimgartner, in Vorbereitung.

[3] H. Behringer, D. Bender, J. Falkenberg \& R. Wiedenmann, Chem. Ber. 101, 1428 (1968).

[4] B. R. O'Connor \& F. N. Jones, J. Org. Chem. 35, 2002 (1970).

[5] M. Stavaux, Bull. Soc. Chim. Fr. 1971, 4426.

[6] D. B. Easton, D. Leaver \& T.J. Rawlings, J.C. S., Perkin I 1972, 41.

[7] C. Gueden \& J. Vialle, Bull. Soc. Chim. Fr. 1973, 270.

[8] M.S. Chauhan, M.E. Hassan \& D. M. McKinnon, Can. J. Chem. 52, 1738 (1974).

[9] V.N. Drozd, Yu.M. Udachin, G.S. Bogomolova \& V.V. Sergeichuk, J. Org. Chem. USSR 16, 775 (1980).

[10] T. Nishiwaki, E. Kawamura, N. Abe, H. Kochi, Y. Sasaoka \& K. Soneda, Heterocycles 16, 595 (1981).

[11] D. Noël \& J. Vialle, Bull. Soc. Chim. Fr. 1967, 2239.

[12] S.V. Amosova, N.N. Skatova, O.A. Tarasova \& B.A. Trofimov, J. Org. Chem. USSR 15, 1841 (1979); siehe auch S. Lahiri, V. Dabral, V. Bhat, E.D. Jemmis \& M. V. George, Proc. Indian Acad. Sci. 86A, 1 (1977). 